\title{
Investigation of the 'cuff' method for assessing seizure duration in electroconvulsive therapy
}

\author{
AIMS AND METHOD \\ Electroconvulsive therapy should be \\ effectively monitored and seizure \\ duration accurately ascertained. We \\ observed the seizure duration in both \\ lower and upper limbs using the \\ Hamilton 'cuff' method.
}

\author{
RESULTS \\ Thirty-nine seizures in 19 patients \\ were observed. There were no statis- \\ tically significant differences \\ between cuffed and uncuffed limbs, \\ nor between upper and lower limbs.
}

\author{
CLINICAL IMPLICATIONS \\ Alternatives to the Hamilton cuff \\ method to observe seizure duration \\ need to be considered.
}

Despite a high stimulus dose a tonic-clonic seizure may not be witnessed. This has been reported to be due to inattention by the attendants, absence of seizure activity or too much muscle relaxation (Pippard \& Ellem, 1980; Fink, 1983). While continuous electroencephalogram (EEG) monitoring has been proposed for quite some time (Fink \& Johnson, 1982; Chesen, 1983) the Royal College of Psychiatrists still recommends the Hamilton cuff technique in its absence (Royal College of Psychiatrists, 1995). With this technique a sphygmomanometer cuff is applied to the contralateral side to the anaesthetic administration site and inflated to above systolic blood pressure, prior to administration of the muscle relaxant. In theory, this should prevent paralysis of distal limb musculature and allows the attendants to observe any seizure activity undiminished by muscle relaxants. We attempted to investigate whether there was any observable difference in observed seizure duration between a 'cuffed' and an 'uncuffed' limb.

\section{Method}

Nineteen patients receiving electroconvulsive therapy (ECT) within a six-month period gave consent to be included in the trial. Patients with peripheral vascular disease were excluded, as were patients who could not read English. Standard ECT procedures were followed. After administration of the anaesthetic agent, arm and leg cuffs, contralaterally placed, were inflated to $200 \mathrm{~mm} \mathrm{Hg}$ and $220 \mathrm{~mm} \mathrm{Hg}$ respectively. A specific leg cuff was used to prevent erroneously elevated sphygmomanometer readings. The muscle relaxant followed by the stimulus dose was then administered. Three observers noted the duration of the convulsion: one observed the face, one the arms and one the legs. Seizure duration was defined as the length of time between cessation of the stimulus and absence of tonic-clonic seizures

\section{Results}

Thirty-nine convulsive episodes were observed. The mean seizure duration (MSD) for the head was 23.9 seconds (s.d. =8.8). In the upper limbs, the MSD was 24.2 (s.d.=8.95) seconds cuffed and 23.6 seconds uncuffed (s.d.=9.5). In the lower limbs, the MSD was 25.1 seconds cuffed (s.d.=8.6) and 25.9 seconds (s.d.=8.2) uncuffed. Data were analysed using the Statistical Package for Social Sciences v7.5 for Windows. A two-way analysis of variance (ANOVA), the appropriate test for simultaneous continuous observations, found no significant difference between upper and lower limbs, nor between cuffed and uncuffed limbs in terms of the MSD. In no case did a seizure occur in a cuffed limb and not in the uncuffed limb.

\section{Comments}

From these results it appears that there is little observable difference between a cuffed and an uncuffed limb, even if lower limbs and specialist tourniquets are used. Several authors have suggested that there is little difference in seizure duration observed with EEG monitoring and the Hamilton 'cuff' method (Lambert \& Petty, 1994). Our data suggests that there is no difference between a cuffed limb and an uncuffed limb. We suggest that the use of the Hamilton cuff method to 'observe' absent seizures should cease; this merely delays addressing the real cause of 'absent' seizures and may involve anaesthesia without therapeutic effect. This recommendation should be dropped from the Royal College of Psychiatrists (1995) handbook on ECT. Given that as long ago as 1966 the literature suggested the adoption of continuous EEG monitoring, and that the Hamilton cuff method has been shown to be ineffective, the adoption of EEG monitoring as standard needs to be seriously considered.

\section{References}

CHESEN, E. S. (1983) EEG Monitoring of ECT preferred to cuff method. American Journal of Psychiatry, 140, 1648-1649.

FINK, M. (1983) Missed seizures and the bilateral-unilateral electroconvulsive therapy controversy. American Journal of Psychiatry, 140, 1198-1199.

- \& JOHNSON, L. Monitoring the duration of electroconvulsive therapy seizures: 'cuff' and EEG methods compared. Archives of General Psychiatry, 39, 1189-1191.

LAMBERT, M. \& PETTY, F. (1994) EEG seizure duration monitoring of ECT. Progress in Neuropsychopharmacology \& Biological Psychiatry, 18, 497-502.

PIPPARD, J. \& ELLAM, L. (1980) ElectroconvulsiveTreatment in Great Britain, 1980. London: Headley Brothers.

ROYAL COLLEGE OF PSYCHIATRISTS (1995) The ECT Handbook. Second Report of the Royal College of Psychiatrists'special Committee on ECT. London: Gaskell.

*M. E. Jan Wise Registrar in Psychiatry, Fiona Mackie Senior House Officer in Psychiatry, Antonios C. Zamar Lecturer and Honorary Senior Registrar in General Adult Psychiatry, James P. Watson Professor of Psychiatry, Guy's, King's \& StThomas, Department of Psychiatry, Thomas Guy House, Guy's Hospital, London SE1 9RT 